\title{
Drogas, Trastorno por Uso de Sustancias y Conducción: La intervención de los profesionales que trabajan en adicciones
}

\section{Drugs, Substance Use Disorder and Driving: Intervention of Health Professionals in the Treatment of Addictions}

\author{
Francisco Javier Álvarez*, Juan Carlos González-Luque**, María Seguí-Gómez** \\ *Farmacología, Facultad de Medicina, Universidad de Valladolid. **Dirección General de Tráfico, Madrid.
}

$\mathrm{Y}$ a no hay dudas: conducir con la presencia de drogas es un problema real, se asocia a un mayor riesgo de implicación en colisiones de tráfico, y la intervención dirigida a evitar conducir con la presencia de drogas constituye una prioridad (Álvarez y González-Luque, 2010; DRUID, 2012; Schulze et al., 2012).

En este artículo se utiliza el concepto de lesiones por colisiones de tráfico y no el término inadecuado de accidentes de tráfico. La gran mayoría de la lesiones por colisiones de tráfico son evitables (Álvarez, 2005; Redelmeier y McLellan, 2013). De ahí el interés de esta editorial, sensibilizar a los profesionales sanitarios, y en particular a los que tratan a pacientes con trastorno por uso de sustancias (TUS), acerca de que pueden y deben intervenir en la prevención de las lesiones por colisiones de tráfico, ya que se trata de lesiones evitables, tal como señalaba el eslogan del Día Mundial de la Salud de 2004, "la seguridad vial no es accidental' (Álvarez, 2005).

Cuando se habla de drogas y seguridad vial, sin lugar a dudas, la más peligrosa es el alcohol. De acuerdo a las conclusiones del proyecto europeo DRUID (DRUID, 2012; Schulze et al., 2012), las intervenciones en relación a otras drogas distintas del alcohol no pueden realizarse a costa de reducir las intervenciones en relación al alcohol (Romano,
Torres-Saavedra, Voas y Lacey, 2014). Y ello es aplicable a los profesionales que trabajan en el campo de las adicciones: intervenir en relación al alcohol y las drogas y la conducción, debe realizarse de forma integrada, y en ningún caso dejando de actuar en relación al alcohol.

\section{Sobre la terminología: Conducir con la presencia de drogas}

En este artículo se menciona el término conducir con la presencia de drogas, es decir con cantidades cuantificables de drogas en el organismo, y se evita el término conducir bajo los efectos o influencia de drogas. En algunos países se utiliza el criterio de tolerancia cero: es decir, cuando cualquier nivel de droga en el organismo es sancionable, o si se establece una determinada concentración en el organismo, o punto de corte, por encima del cual es sancionable conducir. En otros casos se habla de deterioro, cuando lo que no está permitido, y por tanto es sancionable, es el hecho de que el conductor muestre indicios de deterioro (conducción bajo la influencia o bajo los efectos), lo que se suele evidenciar mediante la utilización de diversas pruebas de campo (pruebas de coordinación... etc.) (Álvarez y González-Luque, 2014). 


\section{Conducir con la presencia de drogas: ¿un problema real?}

Diversos estudios previos han mostrado que conducir con presencia de drogas es frecuente (Barlés, Escario y Galbé, 2014; EMCDDA, 2007; Verstraete y Legrand, 2014), si bien los resultados deben analizarse teniendo en cuenta el tipo de población de conductores incluida (población general, infractores, lesionados o fallecidos).

El proyecto europeo DRUID (DRUID, 2012; Schulze et al., 2012) ha aportado datos a nivel europeo, obtenidos en controles de carretera realizados al azar: en el $8.43 \%$ (rango: $1.34-15.01 \%$ ) de los conductores se confirmó la presencia de alcohol/drogas/ciertos medicamentos, existiendo grandes diferencias entre países: en Italia $(15.01 \%)$ y en España $(14.85 \%)$ fueron los países donde más frecuentemente se encontraron conductores positivos en alguna sustancia. A nivel Europeo, la droga (sin considerar las asociaciones de sustancias) más frecuentemente detectada fue el alcohol (3.48\%), seguida del cannabis (THC) (1.32\%) y cocaína $(0.42 \%)$. La mayor prevalencia de positivos a cannabis y cocaína se observó en conductores españoles. Además, es preciso resaltar que el porcentaje de conductores que conducen con presencia de alguna droga en España $(7.63 \%)$ es el más elevado de todos los países participantes en el estudio.

Según un estudio reciente (Fierro, González-Luque, Seguí-Gomez y Álvarez, 2015), realizado en muestras representativas de conductores de vehículos de España en 2008/9 y 2013, se ha observado un descenso en la frecuencia de conductores con presencia de alcohol (4.92\% [IC 95\%, $4.18-5.66$ ] en $2008 / 9$ y $3.41 \%$ [2.27-4.07] en 2013) y drogas $(6.93 \%$ [6.07-7.80] en $2008 / 9$ y $4.87 \%$ [4.09-5.65] en 2013). El descenso en los casos positivos a drogas se ha debido en gran medida a un descenso en los casos positivos a cannabis. El uso rutinario de controles de drogas en carretera podría haber sido un factor contribuyente a ese descenso observado.

\section{Drogas y riesgo de accidentabilidad}

En la actualidad el desarrollo de intervenciones preventivas ha de basarse en la evidencia científica. Por ello una de las prioridades del proyecto Europeo DRUID fue analizar el riesgo de accidentalidad (lesiones graves o muerte) de conducir con la presencia de drogas (DRUID, 2012; Schulze et al., 2012).

Para la interpretación de los resultados en el proyecto DRUID se establecieron 4 niveles de riesgo:

Ligero incremento del riesgo ( $R R=1-3)$ : Riesgo asociado a la conducción con presencia de alcohol (en sangre) entre 0.1 $\mathrm{y}<0.5 \mathrm{~g} / \mathrm{L}, \mathrm{y}$ a conducir con presencia de cannabis.

Incremento medio del riesgo $(R R=2-10)$ : Para la conducción con una concentración de alcohol (en sangre) entre 0.5 $\mathrm{g} / \mathrm{L}$ y $<0.8 \mathrm{~g} / \mathrm{L}$, o con la presencia de cocaína, opiáceos ilegales y medicinales, benzodiacepinas y Z-hipnóticos (sin mezclar unos grupos de sustancias con otros).

Incremento alto del riesgo $(R R=5-30)$ : Conducción con presencia de alcohol (en sangre) entre $0.8 \mathrm{y}<1.2 \mathrm{~g} / \mathrm{L}$, así como con la presencia de anfetaminas y con la mezcla de diferentes drogas.

Incremento extremadamente alto del riesgo $(R R=20-200)$ : Conducción con presencia de alcohol (en sangre) $\geq 1.2 \mathrm{~g} / \mathrm{L}, \mathrm{y}$ con la presencia de alcohol y drogas simultáneamente.

Por tanto, los datos muestran que el alcohol $(\geq 0.8 \mathrm{~g} / \mathrm{L})$, y la combinación o policonsumo (alcohol+drogas o asociación de drogas), se asocian al mayor riesgo de colisión de tráfico.

En el caso del cannabis es sobre el que mayor información disponemos, y sobre el que se podría establecer una relación entre concentración y riesgo (Asbridge, Hayden y Cartwright, 2012; Laumon et al., 2006; Verstraete y Legrand, 2014). Recientemente Elvik (2013) y Verstraete y Legrand (2014) han revisado la información de que disponemos en relación al alcohol, drogas y ciertos tipos de medicamentos y colisiones de tráfico.

\section{Conducir con presencia de drogas: aspectos legislativos}

En la mayoría de países esta sancionada la conducción con presencia de drogas (EMCDDA, 2015). Si analizamos el caso de España, estamos ante una materia regulada tanto como infracción en el ámbito administrativo, como delito en el ámbito penal, si bien nunca pueden concurrir ambas sanciones simultáneamente. El modelo pasa por una diferenciación entre la "presencia" y el peligro abstracto en las leyes administrativas (criterio de tolerancia cero) y la "influencia" (criterio de deterioro) y el peligro concreto del Código Penal (Álvarez y González-Luque, 2014).

Recientemente la legislación Española ha introducido cambios relevantes (Ley 6/2014) acerca de:

- La prohibición de la conducción con la presencia de drogas en el organismo; es decir, el principio de "tolerancia cero" en materia de drogas y conducción.

- La consideración de la prueba salival, mediante dispositivo autorizado, como medio preferente para detectar in situ la presencia de drogas en el organismo y como medio electivo para su posterior análisis de confirmación.

- La sanción por conducir con presencia de drogas en el organismo o por negarse a realizar las pruebas para su detección, se establece en $1.000 €$, además de la pérdida de 6 puntos del carnet de conducir.

\section{La realización de pruebas en carretera para la detección de drogas: saliva versus sangre}

Hasta ahora, y debido a las dificultades jurídicas y prácticas para la extracción de sangre, era poco frecuente que se 
realizasen pruebas en carretera para detectar la presencia de drogas en el conductor. En la actualidad, sin embargo, es posible determinar las drogas en saliva, más específicamente en "fluido oral", mediante métodos no cruentos ni invasivos. Estos sistemas ya están disponibles y se utilizan de forma rutinaria. Aunque existen ciertas limitaciones, en especial en relación con la sensibilidad, especificidad y puntos de corte (Verstraete, 2005), la detección de drogas en fluido oral es garantista cuando el análisis en carretera se asocia a un posterior análisis de confirmación en laboratorio, como es el caso de la regulación legal de España.

En la prueba en carretera se utilizan distintos dispositivos. En el caso de España, en la actualidad, se están utilizando Dräger DrugTest ${ }^{\circledR} 5000$, DrugWipe ${ }^{\circledR}$, Alere $^{\mathrm{TM}}$ DDS $^{\circledR 2}$ Mobile Test System. Con ellos se pueden detectar diversos grupos de sustancias: cannabis, opiáceos, cocaína, anfetamina y análogos, benzodiacepinas, etc. Es preciso señalar que estos dispositivos detectan en fluido oral la sustancia activa lo que supone la constatación de consumo reciente.

En el proyecto DRUID se estableció el listado de sustancias a detectar (27) y sus puntos de cortes analíticos para la prueba de confirmación y cuantificación por cromatografía, con el fin de su uso para estudios epidemiológicos. Es preciso señalar que la información de que se dispone sobre la equivalencia entre sangre y saliva (fluido oral) es limitada, si bien la utilizada en el DRUID se considera en la actualidad como de referencia (DRUID, 2012; Schulze et al., 2012). Recientemente se han propuesto equivalencias entre sangre y fluido oral para 12 sustancias (Gjerde, Langel, Favretto y Verstraete, 2014).

La realización en conductores de pruebas de drogas en fluido oral es ya una situación sin retorno en los países desarrollados.

\section{¿Qué podemos hacer los profesionales sanitarios?}

Hasta ahora nos hemos referido en general a los conductores, y a conducir con la presencia de drogas. Sin embargo, los profesionales que tratan las adicciones atienden a los pacientes con TUS. ¿Por qué es para estos profesionales la conducción algo importante y relevante en su práctica clínica? y ¿porque deben de intervenir activamente?

\section{1. ¿Conducen nuestros pacientes con TUS?}

Estudios previos (Álvarez, Gómez-Talegón y Marcos, 2010) ya sugerían que estos pacientes conducían con frecuencia. Pero han sido los datos del estudio multicéntrico español PROTEUS (Roncero et al., 2013) los que lo han confirmado: una parte relevante de los pacientes dependientes de opiáceos en tratamiento conduce (52\%). El 94\% de los pacientes estaban en tratamiento con metadona, con una dosis media de $60 \mathrm{mg} /$ día; a esta dosis la metadona interfiere, o puede interferir, con la conducción. Los pacien- tes que conducían presentaban menos problemas legales. Ello podría ser debido a que el hecho de conducir vehículos podría ser un factor de "normalización" para el paciente, siendo necesario en algunos casos para su actividad profesional. Es por ello que se debe prestar atención a que estos pacientes puedan conducir con seguridad.

\section{Los pacientes con TUS, déficits neuropsicológi- cos y aptitud para conducir}

El uso continuado de drogas se asocia con déficits en varios dominios o campos neuropsicológicos. Por otra parte, los pacientes con TUS presentan frecuente comorbilidad orgánica y frecuente patología dual. Finalmente, los pacientes con TUS reciben tratamiento específico para su adicción, así como tratamiento psicofarmacológico complementario, que puede interferir con el rendimiento psicomotor y una adecuada aptitud para conducir.

Es importante tener en cuenta estos tres aspectos, i) la propia enfermedad, la adicción, y los déficits neuropsicológicos, ii) la comorbilidad-patología dual, y iii) el tratamiento farmacológico en el proceso de tratamiento/reinserción de los pacientes con TUS (Baldaccino et al., 2012; Lorea et al., 2011; Lundqvist, 2005; Soler, Balcells y Gual, 2014).

La normativa europea (CD 439/1991/EEC) y la normativa española (Real Decreto 818/2009) establece en qué circunstancias no se puede otorgar o prorrogar el permiso de conducción. En el caso de España, la valoración de la aptitud para conducir se realiza en los Centros de Reconocimiento de Conductores (CRC). El diagnóstico de un TUS por sí mismo no implica un deterioro de la capacidad de conducir que impida la conducción. En todo caso, la evaluación del conductor con TUS debe hacerse de forma individual en dichos CRC, con la necesaria valoración del informe del facultativo que trate al paciente.

Un aspecto que genera problemas en la práctica clínica es la valoración de la aptitud para conducir y la información a los CRC. En ocasiones el profesional sanitario debe emitir un informe para estos CRC sobre el paciente, bien a petición de este, de los propios CRC o de las autoridades. La función del profesional que atiende a los pacientes con adicciones no es señalar si el paciente puede o no conducir, sino de informar acerca de la situación clínica del paciente. El informe de valoración debe contener: diagnóstico, cumplimiento y adherencia al tratamiento, fechas de remisiones o recaídas, conducta y riesgo suicida, tiempo desde que está estable, y posibles efectos secundarios de la medicación, así como si consume o no cualquier tipo de drogas (por ejemplo, análisis de drogas en orina, etc) (Álvarez y González-Luque, 2014).

Otra cuestión relacionada con la anterior, pero independiente, es el consejo médico que se debe ofrecer al paciente en cada visita, y en particular si debe o no conducir, o limitar sus actividades, en base a su situación clínica. Estos aspectos serán analizados con mayor detalle en apartado 6. 


\section{3. ¿Tienen un mayor riesgo de colisiones de trafico los pacientes con TUS?}

Los conductores con determinadas patologías psiquiátricas presentan un mayor riesgo de colisión de tráfico que los conductores sanos. En un metanálisis (Vaa, 2003) en el que se analiza el riesgo de colisión de tráfico en relación a los procesos orgánicos y mentales señalados en la Directiva Europea CD 91/439/EEC, los trastornos mentales son una de las condiciones que presentan mayor riesgo de implicación en colisión de tráfico ( $R R=1,72$, IC 95\%, 1,48-1,99), junto con el abuso o dependencia del alcohol $(\mathrm{RR}=2,00$, IC $95 \%$, $1,89-2,12)$ y el abuso o dependencia de drogas $(\mathrm{RR}=1,58$, IC 95\%, 1,45-1,73).

Por otra parte, diversos estudios (Álvarez, Gómez-Talegón y Marcos, 2010; Gomes et al., 2013) evidencian que los pacientes con TUS presentan una elevada accidentalidad, no sólo colisiones de tráfico, y que dichas colisiones de tráfico contribuyen al exceso de mortalidad de estos pacientes, aun cuando esta no sea la principal causa de mortalidad (Degenhardt el al., 2014).

\section{La prescripción de fármacos al paciente con TUS $y$ conductor}

El empleo de fármacos para tratar la patología de base (TUS), patología dual y comorbilidad orgánica, hace que la prescripción fármacos sea frecuente en estos pacientes, como ya se ha comentado.

De los fármacos autorizados para el tratamiento de las adicciones, bupropion, vareclidina, naltrexona, buprenorfina y metadona incluyen el pictograma de medicamentos y conducción en España (Fierro, Gómez-Talegón y Álvarez, 2013), mientras que no lo poseen la nicotina, ni el acamprosato, carbimida, disulfiramo y nalmefeno (AEMPS, 2015; Álvarez y González-Luque, 2014). Por otra parte, la ficha técnica y el prospecto incluye información sobre el efecto de la medicación en la conducción (Ravera et al., 2012).

Aunque el citado pictograma solo tiene carácter informativo, se debe prestar atención a este hecho e informar siempre al paciente. En especial cuando la mayoría de los pacientes con TUS van a estar en tratamiento con psicofármacos: de los 198 principios activos autorizados del grupo $\mathrm{N}$ en España, 180 llevan pictograma (AEMPS, 2015; Álvarez y González-Luque, 2014).

Recientemente ha sido objeto de atención los pacientes en programas de mantenimiento con agonistas opiáceos (metadona y buprenorfina) y la conducción de vehículos (Strand, Fjeld, Arnestad y Morland, 2013; Soyka, 2014). Ambos fármacos pueden afectar a la capacidad para conducir con seguridad, de ello se informa en la ficha técnica, prospecto, y tienen el pictograma de medicamentos y conducción de vehículos en España. Se han señalado algunas ventajas de la buprenorfina (Roncero et al., 2013) en el sentido de que deterioraría menos el rendimiento psicomotor en pacientes con TUS por opiáceos que la metadona (Rapeli, Fabritius, Kalska y Alho, 2011; 2012).

El factor clave es la patología, los déficits neuropsicológicos y la comorbilidad médica y psiquiátrica. La medicación es un factor añadido: esta puede interferir negativamente al principio del tratamiento, pero a medida que el paciente mejora en su situación clínica el efecto es positivo. Se debe prestar especial atención a la prescripción de fármacos en estos pacientes y la posibilidad de interacciones que aumenten en especial los efectos sedantes sobre el SNC, efectos anticolinérgicos y efectos sobre la visión, y debe evitarse el consumo de alcohol y otras drogas (Álvarez y González-Luque, 2014).

Los profesionales sanitarios deben conocer e informar a sus pacientes, especialmente en el campo de las adicciones, acerca de que cierta medicación (opiáceos, benzodiacepinas...) podría dar positivo en los controles de drogas en carretera.

\section{El tratamiento reduce la siniestralidad por tráfi- co de los pacientes con TUS}

Cada vez existe mayor evidencia de que el hecho de que el paciente con TUS esté en un programa de tratamiento contribuye a reducir su implicación en colisiones de tráfico (Darke et al., 2004; Gómez-Talegón y Álvarez, 2006).

En un estudio Noruego (Bukten et al., 2013), con un seguimiento de 9 años y que recoge los datos de colisiones e infracciones de tráfico de pacientes en tratamiento de sustitución de opiáceos, antes, durante y después el tratamiento, se observó que en los pacientes en tratamiento de sustitución de opiáceos se reducían los colisiones e infracciones de tráfico en un $40 \%$.

\section{Drogas, adicciones y conducción de vehículos: la información y los consejos al paciente con TUS}

A continuación presentamos los puntos clave, en nuestra opinión, sobre la información y el consejo médico/sanitario a los pacientes con TUS.

- En base a la evidencia científica en pacientes con TUS y la conducción de vehículos (cambios de conducta al volante y agresividad vial (Benavidez, Flores, Fierro y Álvarez, 2013), frecuente implicación en colisión de tráfico de tráfico y otro tipo de accidentes (Álvarez, Gómez-Talegón y Marcos, 2010; Coghlan y Macdonald, 2010; Darke, Kelly y Ross, 2004; Macdonald et al., 2004), mayor riesgo de accidentalidad de tráfico (Schulze et al., 2012; Verstraete y Legrand, 2014)), se debe informar a los pacientes de que si conducen con la presencia de drogas están adoptando una conducta de riesgo para ellos y para el resto de usuarios de las vías (Álvarez y González-Luque, 2010; 2014).

- Se debe informar a los pacientes de que la enfermedad, comorbilidad, etc, y los efectos de la medicación puedan afectar su aptitud para conducir con seguridad. En este sentido, se ha evidenciado que el consejo médico a 
pacientes que potencialmente no eran aptos para conducir, contribuía a una reducción en las colisiones por tráfico (Redelmeier et al., 2012).

- Prescribir la medicación que menos interfiere con el rendimiento psicomotor es prioritario. Evitar las interacciones farmacodinamicas que potencien los efectos sedantes, anticolinérgicos y sobre la visión es importante. Para ello, además, se debe informar al paciente de que no puede consumir alcohol y/o drogas, no solo para no conducir con presencia de drogas, sino además porque así se evitan posibles interacciones medicamentosas.

- Deben ser informados de que el tratamiento no solo mejora su proceso patológico, sino que se asocia a una menor implicación en colisiones de tráfico.

- Además, deberían ser informados de que los dispositivos actuales detectan la presencia de drogas y ciertos medicamentos (opiáceos, benzodiacepinas...) en saliva, y que serán en su caso sancionados. En España se prevé una futura regulación que evite esta sanción si están siendo controlados medicamente y cumplen determinadas criterios.

Un aspecto importante es el consejo o recomendación de conducir o no: Sin lugar a dudas, los momentos más críticos son al inicio del tratamiento. Se debe recomendar a los pacientes no conducir o limitar al máximo (trayectos cortos, descansar frecuentemente..) el conducir. Esta recomendación deberá actualizarse en cada visita al principio del tratamiento según la evolución clínica de cada paciente. Una adecuada información permitirá al paciente ser consciente del riesgo vial para él y el resto de usuarios de las vías.

Otro aspecto es si el paciente tiene o no la adecuada aptitud para conducir (véase punto 2). Ello debe ser evaluado por el organismo competente, en el caso de España en los Centros de Reconocimiento de Conductores. Pero en cualquier caso no evita que como profesionales sanitarios informemos al paciente con TUS y le recomendemos que no conduzca, ni realice otras actividades de riesgo, cuando procede.

Se ha elaborado un tríptico con algunos de los aspectos que los pacientes con TUS deben conocer sobre drogas y conducción (Álvarez y González-Luque, 2014).

\section{Conclusión}

Conducir vehículos es un buen factor de pronóstico para la integración social del paciente con TUS y se deben desarrollar intervenciones para su implementación, permitiendo o favoreciendo que estos pacientes conduzcan con seguridad bajo supervisión médico-psicológica. Los profesionales sanitarios deben implicarse activamente, informando y aconsejando a sus pacientes (Redelmeier y Tien, 2014) así como seleccionando adecuadamente la medicación a prescribir.

\section{Conflicto de interés}

Los autores declaran que no existe conflicto de interés.

\section{Financiación}

La realización de este trabajo (F. Javier Álvarez) ha sido realizada a través de la participación en la Red Temática de Trastornos Adictivos, Instituto de Salud Carlos III, Ministerio de Economía y Competitividad, Red de Trastornos Adictivos nodo RD12/0028/0012.

\section{Referencias}

AEMPS (2015). Medicamentos y conducción. Recuperado de http://www.aemps.gob.es/industria/etiquetado/ conduccion/home.htm

Álvarez, F. J. (2005). La prevención de las lesiones derivadas de las colisiones de tráfico. Semergen - Medicina de Familia, 31, 151-153. doi:10.1016/S1138-3593(05)72904-X.

Álvarez, F. J., Gómez-Talegón, T. y Marcos, A. (2010). Accident rates for drug-dependent patients in treatment for substance dependence: a pilot trial. Traffic Injury Prevention, 11, 460-465. doi:10.1080/15389588.2010.49 2844.

Álvarez, F. J. y González-Luque, J. C. (2010). Illicit drugs and driving. Medicina Clinica (Barc), 135, 549-551. doi:10.1016/j.medcli.2009.05.033.

Álvarez, F. J. y González-Luque, J. C. (2014). Drogas, adicciones y aptitud para conducir. $3^{\text {a }}$ Ed. Valladolid: Universidad de Valladolid. Recuperado de http://www. drogasyconduccion.com/otros.php?seccion=3

Asbridge, M., Hayden, J. A. y Cartwright, J. L. (2012). Acute cannabis consumption and motor vehicle collision risk: systematic review of observational studies and metaanalysis. British Medical Journal, 344, e536. doi:10.1136/ bmj.e536

Baldacchino, A., Balfour, D. J., Passetti, F., Humphris, G. y Matthews, K. (2012). Neuropsychological consequences of chronic opioid use: a quantitative review and metaanalysis. Neuroscience $\mathcal{E}$ Biobehavioral Reviews, 36, 20562068. doi:10.1016/j.neubiorev.2012.06.006

Barlés Arizon, M.J., Escario, J.J. y Galbé Sánchez-Ventura, J. (2014). Predictors of driving under the influence of alcohol among Spanish adolescents. Adicciones, 26, 96105.

Benavidez, D. C., Flores, A. M., Fierro, I. y Álvarez, F. J. (2013). Road rage among drug dependent patients. Accident Analysis E Prevention, 50, 848-853. doi:10.1016/j. aap.2012.07.010.

Bukten, A., Herskedal, A., Skurtveit, S., Bramness, J. G. y Clausen, T. (2013). Driving under the influence (DUI) among patients in opioid maintenance treatment (OMT): a registry-based national cohort study. Addiction, 108, 1954-1961. doi:10.1111/add.12275. 
Coghlan, M. y Macdonald, S. (2010). The role of substance use and psychosocial characteristics in explaining unintentional injuries. Accident Analysis E Prevention, 42, 476-479. doi:10.1016/j.aap.2009.09.010

Council Directive 91/439/EEC of 29 July 1991 on driving licences. Official Journal, 1991; L 237: 0001-0024. Recuperado de http:/ / eur-lex.europa.eu/legal-content/ES/ TXT/?uri=CELEX:31991L0439

Darke, S., Kelly, E. y Ross, J. (2004). Drug driving among injecting drug users in Sydney, Australia: Prevalence, risk factors and risk perceptions. Addiction, 99, 175-185. doi:10.1046/j.1360-0443.2003.00604.x

Degenhart, L., Larney, S., Randall, D., Burns, L. y Hall, W. (2014). Causes of death in a cohort treated for opioid dependence between 1985 and 2005. Addiction, 109, 9099. doi:10.1111/add.12337

DRUID (2012). Final Report: Work performed, main results and recommendations. Revisión 2.0. Cologne: BAST. Recuperado de http://www.druid-project.eu/ Druid/EN/Dissemination/downloads_and_links/Final_Report.html?nn=613800

Elvik, R. (2013). Risk of road accident associated with the use of drugs: a systematic review and meta-analysis of evidence from epidemiological studies. Accident Analysis $\mathcal{E}$ Prevention, 60, 254-267. doi:10.1016/j. aap.2012.06.017.

EMCDDA (2015). Legal approaches to drugs and driving. Recuperado de http://www.emcdda.europa.eu/html. cfm/index19034EN.html.

EMCDDA (2007). Drugs and Driving. Luxembourg: Office for Official Publications of the European Communities, 2007. Recuperado de http://www.drugsandalcohol. ie/6368/1/EMCDDA_Selected_issue_2007_Driving. pdf

Fierro, I., Gómez-Talegón, T. y Álvarez, F.J. (2013). The Spanish pictogram on medicines and driving: The population's comprehension of and attitudes towards its use on medication packaging. Accident Analysis E Prevention, 50, 1056-1061. doi: 10.1016/j. aap.2012.08.009.

Fierro, I, González-Luque, J.C., Seguí-Gómez, M. y Álvarez, F.J. (2015). Alcohol and drug use by Spanish drivers: Comparison of two cross-sectional road-side surveys (2008-9/2013). International Journal of Drug Policy, 26, 794-797, doi:10.1016/j.drugpo.2015.04.021.

Gjerde, H., Langel K., Favretto, D. y Verstraete, A.G. (2014). Estimation of equivalent cutoff thresholds in blood and oral fluid for drug prevalence studies. Journal of Analytical Toxicology, 38, 92-98. doi:10.1093/jat/bkt122.

Gomes, T., Redelmeier, D. A., Juurlink, D. N., Dhalla, I. A., Camacho, X. y Mamdani M. M. (2013). Opioid dose and risk of road trauma in Canada: a population-based study. JAMA Internal Medicine, 173, 196-201. doi:10.1001/2013. jamainternmed.733
Gómez-Talegón, M. T. y Álvarez, F. J. (2006). Road traffic accidents among alcohol-dependent patients: the effect of treatment. Accident Analysis E Prevention, 38, 201-217. doi:10.1016/j.aap.2005.09.006

Laumon, B., Gadegbeku, B., Martin, J. L., Biecheler, M. B. SAM Group (2006). Cannabis intoxication and fatal road crashes in France: population based case-control study. British Medical Journal, 332, 1298. doi:10.1136/ bmj.332.7553.1298

Ley $6 / 2014$, de 7 de abril, por la que se modifica el texto articulado de la Ley sobre Tráfico, Circulación de Vehículos a Motor y Seguridad Vial, aprobado por el Real Decreto Legislativo 339/1990, de 2 de marzo. Recuperado de http://www.boe.es/diario_boe/txt.php?id=BOE-A-2014-3715

Lorea, I., Fernández-Montalvo, J., Tirapu-Ustárroz, J., Landa, N. y López-Goñi, J.J. (2010) . Neuropsychological performance in cocaine addiction: a critical review. Revista de Neurología, 51, 412-426.

Lundqvist, T. (2005). Cognitive consequences of cannabis use: comparison with abuse of stimulants and heroin with regard to attention, memory and executive functions. Pharmacology Biochemistry $\mathcal{E}$ Behavior, 81, 319-330. doi:10.1016/j.pbb.2005.02.017

Macdonald, S., Mann, R. E., Chipman, M. y Anglin-Bodrug, K. (2004). Collisions and traffic violations of alcohol, cannabis and cocaine abuse clients before and after treatment. Accident Analysis and Prevention, 36, 795-800. doi:10.1016/j.aap.2003.07.004

Ravera, S., Monteiro, S., de Gier J. J., van der Linden, T., Gómez-Talegón, T., Álvarez, F.J.y the DRUID Project WP4 Partner (2012). A European approach to categorising medicines for fitness to drive: Outcomes of the DRUID project. British Journal of Clinical Pharmacology, 74, 920931. doi:10.1111/j.1365-2125.2012.04279.x.

Rapeli, P., Fabritius, C., Kalska, H. y Alho, H. (2011). Cognitive functioning in opioid-dependent patients treated with buprenorphine, methadone, and other psychoactive medications: stability and correlates. $B M C$ Clinical Pharmacology, 21, 11-13. doi:10.1186/1472-690411-13.

Rapeli, P., Fabritius, C., Kalska, H. y Alho, H. (2012). Do drug treatment variables predict cognitive performance in multidrug-treated opioid-dependent patients? A regression analysis study. Substance Abuse Treatment, Prevention, and Policy, 2, 7-45. doi:10.1186/1747-597X-7-45.

Real Decreto $818 / 2009$, de 8 de mayo, por el que se aprueba el Reglamento General de Conductores. Recuperado de http://www.boe.es/boe/dias/2009/06/08/pdfs/ BOE-A-2009-9481.pdf

Redelmeier, D. A., Yarnell, C. J., Thiruchelvam, D. y Tibshirani, R. J. (2012). Physicians' warnings for unfit drivers and the risk of trauma from road crashes. 
The New England Journal of Medicine, 367, 1228-1236. doi:10.1056/NEJMsa1114310

Redelmeier, D. A. y McLellan, B. A. (2013). Modern Medicine Is Neglecting Road Traffic Crashes. PLoS Medicine, 10, e1001463. doi:10.1371/journal.pmed.1001463

Redelmeier, D. A. y Tien, H. C. (2014). Medical interventions to reduce motor vehicle collisions. Canadian Medical Association Journal, 186, 118-124. doi:10.1503/cmaj.122001.

Romano, E., Torres-Saavedra, P., Voas, R. B. y Lacey, J. H. (2014). Drugs and alcohol: their relative crash risk. Journal of Studies on Alcohol and Drugs, 75, 56-64.

Roncero, C., Álvarez, J., Barral, C., Gómez-Baeza, S., Gonzalvo, B., Rodríguez-Cintas, L.,... Casas M (2013). Driving and legal status of Spanish opioid-dependent patients. Substance Abuse Treatment, Prevention, and Policy, 8, 19. doi:10.1186/1747-597X-8-19.

Schulze, H., Schumacher, 1. M., Urmeew, R., Auerbach, K., Álvarez, F. J., Bernhoft, I. M.,... Zlender B. (2012). Driving under the influence of drugs, alcohol and medicines in Europe - findings from the DRUID project. European Monitoring Centre for Drugs and Drug Addiction (EMCDDA). Publications Office of the European Union, Luxembourg. doi:10.2810/74023. Recuperado de http://www.emcdda.europa.eu/ attachements.cfm/att_192773_EN_TDXA12006ENN. pdf

Soler González, C., Balcells Oliveró, M. y Gual Solé, A. (2014). Alcohol related brain damage. State of the art and a call for action. Adicciones, 26, 199-207.

Soyka, M. (2014). Opioids and Traffic Safety - focus on Buprenorphine. Pharmacopsychiatry, 47, 7-17. doi:10.1055/s-0033-1358707

Strand, M. C., Fjeld, B., Arnestad, M. y Morland, J. (2013). Can patients receiving opioid maintenance therapy safely drive? A systematic review of epidemiological and experimental studies on driving ability with a focus on concomitant methadone or buprenorphine administration. Traffic Injury Prevention, 14, 26-38. doi:10 $.1080 / 15389588.2012 .689451$.

Vaa, T. (2003). Impairments, diseases, age and their relative risks of accident involvement: Results from meta-analysis. Oslo: Institute of Transport.

Verstraete, A. G. (2005). Oral fluid testing for driving under the influence of drugs: history, recent progress and remaining challenges. European Journal of Forensic Sciences, 50, 143-150. doi:10.1016/j.forsciint.2004.11.023

Verstraete, A. G. y Legrand, S. A. (2014). Drug use, impaired driving and traffic accidents. 2nd Ed. EMCDDA Insights 16. Luxembourg: Office for Official Publications of the European Communities. Recuperado de http://www. emcdda.europa.eu/attachements.cfm/att_229259_EN_ TDXD14016ENN.pdf 